\title{
Linear algebraic theory of partial coherence: discrete fields and measures of partial coherence
}

\author{
Haldun M. Ozaktas \\ Department of Electrical Engineering, Bilkent University, TR-06533 Bilkent, Ankara, Turkey \\ Serdar Yüksel \\ Department of Electrical and Computer Engineering, University of Illinois at Urbana-Champaign, Urbana, \\ Illinois 61801 \\ M. Alper Kutay \\ The Scientific and Technical Research Council of Turkey-UEKAE, Atatürk Bulvarı 221, 06100 Kavaklıdere, \\ Ankara, Turkey
}

Received July 10, 2001; revised manuscript received January 11, 2002; accepted February 20, 2002

\begin{abstract}
A linear algebraic theory of partial coherence is presented that allows precise mathematical definitions of concepts such as coherence and incoherence. This not only provides new perspectives and insights but also allows us to employ the conceptual and algebraic tools of linear algebra in applications. We define several scalar measures of the degree of partial coherence of an optical field that are zero for full incoherence and unity for full coherence. The mathematical definitions are related to our physical understanding of the corresponding concepts by considering them in the context of Young's experiment. (C) 2002 Optical Society of America

OCIS codes: $030.0030,000.5490$.
\end{abstract}

\section{INTRODUCTION}

The theory of partial coherence is a well-established area of optics. ${ }^{1-4}$ In this paper, we will see that formulating the theory in terms of the standard concepts of linear algebra $^{5}$ leads to a number of new perspectives. While not containing any new physics, this approach offers new insights, understanding, and operationality and has the potential to facilitate applications, especially in optical information processing.

We restrict our attention to quasi-monochromatic conditions and do not deal with issues of temporal coherence. We deal with light fields of varying degrees of spatial coherence, as characterized by their mutual intensity (autocorrelation) functions.

In this paper, we consider the case of discrete light fields, since they lead to a particularly simple matrixalgebraic formulation without the distractions accompanying discussions of continuous function spaces. Once the framework is established, it is not difficult to translate the matrix formalism for discrete fields to a continuous formalism. (We indeed present the continuous versions of many results and relations throughout the paper.) It may be argued that dealing with discrete fields is artificial, since optical fields are continuous fields and most discussions of partial coherence are phrased in classical continuous analysis. However, the following points can be raised in response: (i) The discrete formalism presented here captures virtually all of the essential physics while being more transparent and easily comprehensible. (ii) All continuous fields in essence have only a finite number of degrees of freedom and can be represented by finite discrete vectors; in that sense, it can be argued that discrete vectors serve the same representational purpose as that of functions of continuous variables. In fact, some would argue that the discrete representation is superior in that it is less redundant. In any event, discrete and continuous representations are related to each other through sampling and interpolation relations.

In Sections 2 and 3, we define a number of matrices that characterize the second-order correlations of the field. In Section 4, we mathematically define the limits of full coherence and full incoherence in terms of these matrices. In Section 5, we define several measures $c$ of the degree of partial coherence that interpolate between these two extremes, where $c=1$ for full coherence and $c=0$ for full incoherence. Section 6 extends some of these concepts into the spectral domain, whereas Section 7 discusses these concepts in the context of Young's experiment.

\section{MUTUAL INTENSITY MATRIX}

Let $\mathbf{f}=[f(1), f(2), \ldots, f(N)]^{\mathrm{T}}$ be a vector representing a discrete random optical field (mathematically, a finite random sequence). For simplicity, we deal with onedimensional signals, the extension to two dimensions being straightforward. f may be thought to consist of the representative samples of a function of a continuous vari- 
able. Both the spatial extent and the resolution of real systems are finite. Therefore a finite number of samples, taken at intervals determined by Nyquist's sampling theorem, ${ }^{6}$ are sufficient to fully represent continuous signals. The minimum number of samples needed is usually given by the space-bandwidth product or, for the more general case of irregular regions in the spacefrequency plane (phase space), by the space-frequency area. ${ }^{7} \quad$ The discrete vector constituted from these samples has the same number of degrees of freedom as that of the underlying continuous signal; therefore, from an information perspective, both entities are equally good at representing the physical field in question. These issues are particularly carefully discussed in Refs. 8-10. We assume that $N$ is chosen sufficiently large in accordance with Nyquist's theorem so that the discrete vectors have enough degrees of freedom to represent the continuous fields of interest.

We assume quasi-monochromatic conditions and concentrate on the mutual intensity matrix $\mathbf{J}_{\mathbf{f}}$ of $\mathbf{f}$, defined as

$$
\mathbf{J}_{\mathbf{f}}=\left\langle\mathbf{f f}^{\mathrm{H}}\right\rangle \text {, }
$$

where the angle brackets denote ensemble averaging (expectation value) and the superscript $\mathrm{H}$ denotes Hermitian transpose (conjugate transpose). We will simply write $\mathbf{J}$ instead of $\mathbf{J}_{\mathbf{f}}$ when there is no room for confusion. Letting $J(m, n)$ denote the elements of $\mathbf{J}$, we note that $J(n, n)=I(n)$ is the intensity of the field and that $\sum_{k=1}^{N} I(n)$ is the power. We also define a mean-subtracted version of the mutual intensity, denoted $\mathbf{K}_{\mathbf{f}}$ and defined as

$$
\mathbf{K}_{\mathbf{f}}=\left\langle\left(\mathbf{f}-\boldsymbol{\mu}_{\mathbf{f}}\right)\left(\mathbf{f}-\boldsymbol{\mu}_{\mathbf{f}}\right)^{\mathrm{H}}\right\rangle,
$$

where $\boldsymbol{\mu}_{\mathbf{f}}=\langle\mathbf{f}\rangle$ is the mean of $\mathbf{f}$, a vector whose elements are the individual means of the elements of $\mathbf{f}$. Letting $K(m, n)$ denote the elements of $\mathbf{K}$, we note that $K(n, n)$ $=\sigma^{2}(n)$ is the variance of the field. In the language of the theory of random processes or statistics, the mutual intensity matrix $\mathbf{J}$ is an autocorrelation matrix, whereas its mean-subtracted version $\mathbf{K}$ is an autocovariance matrix. The relation between $\mathbf{J}$ and $\mathbf{K}$ is given by $\mathbf{J}=\mathbf{K}$ $+\boldsymbol{\mu}_{\mathrm{f}} \boldsymbol{\mu}_{\mathrm{f}}^{\mathrm{H}}$.

Now the following properties hold for the $\mathbf{J}$ and $\mathbf{K}$ matrices of any field $\mathbf{f}$ (the proofs are omitted where elementary or well-known $\left.{ }^{5,11}\right)$ :

1. $\mathbf{J}$ and $\mathbf{K}$ are Hermitian symmetric: $\quad \mathbf{J}=\mathbf{J}^{\mathrm{H}}$ and $\mathbf{K}=\mathbf{K}^{\mathrm{H}}$.

2. As with all Hermitian symmetric matrices, the eigenvalues of $\mathbf{J}$ and $\mathbf{K}$ are all real.

3. $\mathbf{J}$ and $\mathbf{K}$ are positive semidefinite, and as with all such matrices, the eigenvalues are nonnegative. Furthermore, $|J(m, n)|^{2} \leqslant|J(n, n)||J(m, m)|$, and likewise for $\mathbf{K}$.

4. Eigenvectors corresponding to distinct eigenvalues will always be orthogonal. Furthermore, as with any Hermitian symmetric matrix, a complete set of orthogonal eigenvectors can always be found, even when there are degenerate eigenvalues. Let $\mathbf{U}$ denote the matrix whose columns are the orthonormal eigenvectors. Then this matrix (known as the modal matrix) will diagonalize the Hermitian symmetric matrix $\mathbf{J}$ or $\mathbf{K}$ :

$$
\begin{aligned}
& \mathbf{J}=\mathbf{U}_{\mathbf{J}} \boldsymbol{\Lambda}_{\mathbf{J}} \mathbf{U}_{\mathbf{J}}^{\mathrm{H}}, \\
& \mathbf{K}=\mathbf{U}_{\mathbf{K}} \boldsymbol{\Lambda}_{\mathbf{K}} \mathbf{U}_{\mathbf{K}}^{\mathrm{H}},
\end{aligned}
$$

where $\boldsymbol{\Lambda}_{\mathbf{J}}$ and $\boldsymbol{\Lambda}_{\mathbf{K}}$ are diagonal matrices consisting of the eigenvalues of $\mathbf{J}$ and $\mathbf{K}$ along their diagonals. These expansions are special cases of the singular-value decomposition. ${ }^{5}$ They can also be written in the more explicit form

$$
\begin{gathered}
\mathbf{J}=\sum_{k=1}^{N} \lambda_{\mathbf{J} k} \mathbf{u}_{\mathbf{J} k} \mathbf{u}_{\mathbf{J} k}^{\mathrm{H}}, \\
J(m, n)=\sum_{k=1}^{N} \lambda_{\mathbf{J} k} u_{\mathbf{J} k}(m) u_{\mathbf{J} k}^{*}(n),
\end{gathered}
$$

where $J(m, n)$ denotes the elements of $\mathbf{J}, \lambda_{\mathbf{J} k}$ denotes the $k$ th eigenvalue of $\mathbf{J}$, and $\mathbf{u}_{\mathbf{J k}}(n)$ is the $n$th element of the $k$ th column of $\mathbf{U}_{\mathbf{J}}$. [A similar expression holds for $K(m, n)$, the elements of $\mathbf{K}$.] This expression is sometimes referred to as the spectral expansion of $J(m, n)$ or, since each term in the summation is in the form of an outer product, as an outer-product expansion. In an optical context, this expression is also known as the coherent-mode representation, ${ }^{12,13}$ as will be clear in section 4 .

5. If $\mathbf{J}$ or $\mathbf{K}$ can be expressed as the outer product of two vectors $\mathbf{u}^{\prime}$ and $\mathbf{u}^{\prime \prime}$ in the form $\mathbf{u}^{\prime} \mathbf{u}^{\prime \prime}$, then Hermitian symmetry implies that $\mathbf{u}^{\prime}$ and $\mathbf{u}^{\prime \prime}$ must be parallel, so that by appropriate scaling $\mathbf{J}$ or $\mathbf{K}$ can be expressed in selfouter-product form $\mathbf{u u}^{\mathrm{H}}$. That is, any Hermitian symmetric matrix that can be written in outer-product form can also be written in self-outer-product form.

6. The following are equivalent: (i) $\mathbf{J}$ or $\mathbf{K}$ can be expressed in self-outer-product form (or, by virtue of property 5, merely in outer-product form). (ii) Each row is a multiple of the other rows. (iii) The rank $R$ of the matrix is 1 . (iv) The matrix has only one nonzero eigenvalue [Eq. (6)].

7. More generally, the rank of $\mathbf{J}$ (or $\mathbf{K}$ ) is equal to the number of nonzero eigenvalues. ${ }^{5}$

We will see in Section 4 that matrices satisfying any of the equivalent conditions stated in property 6 above correspond to fully coherent light. However, we will have to define two more matrices before we start discussing coherence and incoherence.

Before continuing with our development, we briefly present continuous versions of some of the results presented above, which follow from an analogous formalism. $^{14}$ If we let $f(x)$ represent a random optical field (mathematically, a random process), the mutual intensity function $J_{f}\left(x_{1}, x_{2}\right)$ of $f(x)$ is defined as

$$
J_{f}\left(x_{1}, x_{2}\right)=\left\langle f\left(x_{1}\right) f^{*}\left(x_{2}\right)\right\rangle .
$$

Again, we will write $J$ instead of $J_{f}$ when no confusion can arise. $J(x, x)=I(x)$ is the intensity. To save space, we will not replicate results for the continuous version $K_{f}\left(x_{1}, x_{2}\right)$ of the matrix $\mathbf{K}_{\mathbf{f}}$. The mutual intensity function is Hermitian symmetric $\left[J\left(x_{1}, x_{2}\right)=J *\left(x_{2}, x_{1}\right)\right]$, and as with all such functions, its eigenvalues are all real. The eigenvalue equation takes the form 


$$
\int_{-\infty}^{\infty} J\left(x, x^{\prime}\right) u_{\nu}\left(x^{\prime}\right) \mathrm{d} x^{\prime}=\lambda_{\nu} u_{\nu}(x),
$$

where $u_{\nu}(x)$ are the eigenfunctions and $\lambda_{\nu}$ are the eigenvalues indexed by $\nu$. The mutual intensity function is also positive semidefinite, and as with all such functions, its eigenvalues are nonnegative. Positive semidefiniteness means that

$$
\int_{-\infty}^{\infty} \int_{-\infty}^{\infty} u^{*}\left(x_{1}\right) J\left(x_{1}, x_{2}\right) u\left(x_{2}\right) \mathrm{d} x_{1} \mathrm{~d} x_{2} \geqslant 0
$$

for any function $u(x)$. As with any Hermitian symmetric function, it is possible to choose the eigenfunctions to be orthonormal even when there are degenerate eigenvalues:

$$
\int_{-\infty}^{\infty} u_{\nu}^{*}(x) u_{\nu^{\prime}}(x) \mathrm{d} x=\delta\left(\nu-\nu^{\prime}\right) .
$$

The continuous counterpart of Eq. (6) is

$$
J\left(x_{1}, x_{2}\right)=\int_{-\infty}^{\infty} \lambda(\nu) u_{\nu}\left(x_{1}\right) u_{\nu}^{*}\left(x_{2}\right) \mathrm{d} \nu,
$$

where $\lambda(\nu)$ is the same as $\lambda_{\nu}$ and we have assumed a continuous eigenvalue spectrum. If $J\left(x_{1}, x_{2}\right)$ can be expressed as the "outer product" of two functions $u^{\prime}\left(x_{1}\right)$ and $u^{\prime \prime}\left(x_{2}\right)$ in the form $u^{\prime}\left(x_{1}\right) u^{\prime \prime *}\left(x_{2}\right)$, then it can also be expressed in self-outer-product form $u\left(x_{1}\right) u^{*}\left(x_{2}\right)$. In a continuous context, being expressible in outer-product form is often referred to as separability. $J\left(x_{1}, x_{2}\right)$ being expressible in self-outer-product form is equivalent to its having a single nonzero eigenvalue: $\lambda(\nu)=\lambda_{0} \delta(\nu$ $-\nu_{0}$ ) for some $\nu_{0}$.

\section{NORMALIZED MUTUAL INTENSITY: COMPLEX COHERENCE MATRIX}

If our interest is more in the relative correlations of various points of the field but not so much in the absolute intensity of the field, then it is more convenient to work with the following normalized versions of $\mathbf{J}$ and $\mathbf{K}$, respectively:

$$
\begin{aligned}
L(m, n) & =\frac{\left\langle f(m) f^{*}(n)\right\rangle}{\left[\left\langle|f(m)|^{2}\right\rangle\left\langle|f(n)|^{2}\right\rangle\right]^{1 / 2}} \\
& =\frac{J(m, n)}{[J(m, m) J(n, n)]^{1 / 2}} \\
M(m, n) & =\frac{\left\langle[f(m)-\mu(m)][f(n)-\mu(n)]^{*}\right\rangle}{\left[\left\langle|f(m)-\mu(m)|^{2}\right\rangle\left\langle|f(n)-\mu(n)|^{2}\right\rangle\right]^{1 / 2}} \\
& =\frac{K(m, n)}{[K(m, m) K(n, n)]^{1 / 2}}
\end{aligned}
$$

where $L(m, n)$ and $M(m, n)$ are the elements of the matrices $\mathbf{L}$ and $\mathbf{M}$, the former of which we refer to as the complex coherence matrix. The diagonal elements of both matrices are identically unity. Note that $\mathbf{M}$ is merely the complex coherence matrix of the mean- subtracted field $\mathbf{f}-\boldsymbol{\mu} . \quad M(m, n)$ is nothing but the standard statistical correlation factor of the two random variables $f(m)$ and $f(n) .{ }^{11}$

Since $\mathbf{L}$ and $\mathbf{M}$ are obtained by normalizing $\mathbf{J}$ and $\mathbf{K}$ in a particular way, they inherit many of their properties:

1. Hermitian symmetry: $\mathbf{L}=\mathbf{L}^{\mathrm{H}}$ and $\mathbf{M}=\mathbf{M}^{\mathrm{H}}$.

2. The eigenvalues of $\mathbf{L}$ and $\mathbf{M}$ are all real.

3. $\mathbf{L}$ and $\mathbf{M}$ are positive semidefinite, and their eigenvalues are nonnegative. Furthermore, $|L(m, n)| \leqslant 1$ and $|M(m, n)| \leqslant 1$.

4. The eigenvectors can be chosen to be orthonormal, and $\mathbf{L}$ and $\mathbf{M}$ can be decomposed as in Eqs. (3) and (5).

5. If $\mathbf{L}$ or $\mathbf{M}$ can be written in outer-product form, it can also be written in self-outer-product form.

6. The following are equivalent: (i) $\mathbf{L}$ or $\mathbf{M}$ can be expressed in self-outer-product form (or in outer-product form). (ii) Each row is a multiple of the other rows. (iii) The rank of the matrix is 1 . (iv) The matrix has only one nonzero eigenvalue.

7. The rank $R$ of $\mathbf{L}$ (or $\mathbf{M}$ ) is equal to the number of nonzero eigenvalues.

Furthermore, we can state the following additional properties:

1. The diagonal entries of both $\mathbf{L}$ and $\mathbf{M}$ are all equal to 1 , corresponding to the fact that each point is by definition fully correlated with itself.

2. If $\mathbf{J}$ (or $\mathbf{K}$ ) has unit rank, then all elements of $\mathbf{L}$ (or M) have unit magnitude. Conversely, if all elements of $\mathbf{L}$ (or M) have unit magnitude, positive semidefiniteness implies that $\mathbf{J}$ (or $\mathbf{K}$ ) has unit rank.

The complex coherence function $L\left(x_{1}, x_{2}\right)$ of a continuous function $f(x)$ is defined as

$$
\begin{aligned}
L\left(x_{1}, x_{2}\right) & =\frac{\left\langle f\left(x_{1}\right) f^{*}\left(x_{2}\right)\right\rangle}{\left[\left\langle\left|f\left(x_{1}\right)\right|^{2}\right\rangle\left\langle\left|f\left(x_{2}\right)\right|^{2}\right\rangle\right]^{1 / 2}} \\
& =\frac{J\left(x_{1}, x_{2}\right)}{\left[J\left(x_{1}, x_{1}\right) J\left(x_{2}, x_{2}\right)\right]^{1 / 2}},
\end{aligned}
$$

whose diagonal elements are identically unity and where $\left|L\left(x_{1}, x_{2}\right)\right| \leqslant 1$. Once again, we will not replicate results for the continuous version $M\left(x_{1}, x_{2}\right)$ of the $\mathbf{M}$ matrix. $L\left(x_{1}, x_{2}\right)$ is Hermitian symmetric and positive semidefinite, and therefore its eigenvalues are real and nonnegative. Just like $J\left(x_{1}, x_{2}\right)$, it can be expanded in terms of its orthonormal eigenfunctions as in Eq. (11). If it can be expressed in outer-product form, then it can also be expressed in self-outer-product form. Again, as with $J\left(x_{1}, x_{2}\right)$, being expressible in self-outer-product form is equivalent to having a single nonzero eigenvalue. In the case of $L\left(x_{1}, x_{2}\right)$, these two conditions are further equivalent to the magnitude of $L\left(x_{1}, x_{2}\right)$ being equal to unity for all $x_{1}$ and $x_{2}$, that is, $\left|L\left(x_{1}, x_{2}\right)\right|=1$.

\section{FULL COHERENCE AND FULL INCOHERENCE}

Full coherence and full incoherence (lack of coherence) are the two extreme cases of the continuum of partial coherence. In most texts, these concepts are introduced 
and discussed for the spatial case through Young's experiment. Under quasi-monochromatic conditions, temporal coherence issues can be set aside, and the interference fringe visibility reflects the degree of coherence of the light. Fully coherent light results in maximum fringe depth (greatest visibility), and fully incoherent light results in zero fringe depth (no visibility). Two samples of a coherent light field result in interference effects, whereas samples of an incoherent field do not. For fully coherent light, we have complex-amplitude superposition, whereas for fully incoherent light we have intensity superposition. The reader is assumed to be already familiar with these elementary concepts. ${ }^{3,15}$

Up to this point, we have introduced four different but closely related matrices that characterize the secondorder correlations of a random optical field and discussed the properties of these matrices, most of which followed from Hermitian symmetry and positive semidefiniteness. Now we will provide a mathematical definition of full spatial coherence and full spatial incoherence in terms of these matrices.

Underlying the concept of coherence or incoherence of a field is the statistical correlation of two spatial samples of that field. In general, a field is considered coherent if any two samples of the field are fully correlated, and a field is considered incoherent if any two distinct samples are fully uncorrelated. Two random variables will be said to be fully correlated if they are just as correlated with each other as they are with themselves. Mathematically, this may be expressed by the requirement that the magnitude of their normalized correlation or covariance have the largest possible value of unity. Two random variables will be said to be fully uncorrelated if the magnitude of their normalized correlation or covariance is as small as possible, namely, zero. In practice, a field can be said to be effectively coherent or incoherent if, for the purposes of the optical system in question, the field behaves as if it were fully coherent or fully incoherent (for instance, based on finite apertures or finite resolution).

Although a number of the results stated below concern the unnormalized matrices $\mathbf{J}$ and $\mathbf{K}$, we will see that working with the normalized matrices $\mathbf{L}$ and $\mathbf{M}$ has certain advantages. However, the choice between $\mathbf{L}$ and its mean-subtracted version $\mathbf{M}$ is not so clear-cut. This depends on precisely how we wish to define coherence and incoherence; we leave this choice of definition to the reader. This issue is further discussed in Section 7 in conjunction with Young's two-slit experiment. For the sake of simplicity in presentation, from now on we work with the mutual intensity matrix $\mathbf{J}$ and the complex coherence matrix $\mathbf{L}$. However, we will keep in mind that we may simply replace $\mathbf{J}$ with $\mathbf{K}$ and $\mathbf{L}$ with $\mathbf{M}$ in the following discussion if this is desirable. Choosing to work with the mean-subtracted version results in a different definition of full coherence and full incoherence, but this difference is not an essential one and the concepts converge to the same physical reality when properly interpreted.

\section{A. Full Incoherence}

First, we consider incoherent fields. Since any two distinct samples of such a field must be uncorrelated, the mutual intensity matrix $\mathbf{J}$ and its normalized version, the complex coherence matrix $\mathbf{L}$, must be diagonal. In fact, as a consequence of normalization, $\mathbf{L}$ is the identity matrix. (Some of the diagonal elements of $\mathbf{J}$ may be zero because the field is zero at those points, in which case the normalization results in 0/0 indefinite entries; however, interpreting the zero elements as the limit of very small elements allows us to consistently maintain that $\mathbf{L}$ is the identity matrix.) Therefore:

A discrete optical field is fully incoherent if the associated normalized mutual intensity matrix (complex coherence matrix) is the identity matrix, i.e.,

$$
\mathbf{L}=\mathbf{I}
$$

or, alternatively, if the associated mutual intensity matrix $\mathbf{J}$ is diagonal.

Though it is trivial, we also note that for the fully incoherent case the matrix $\mathbf{L}$ is of full rank $(R=N)$, and all of its eigenvalues are equal to unity. On the other hand, the $\mathbf{J}$ (or K) matrix for an incoherent field need not be full rank, nor does every full-rank matrix correspond to an incoherent field.

We also note that when the matrix $\mathbf{J}$ is equal to a scalar multiple of the identity matrix, the underlying field $\mathbf{f}$ is stationary and referred to as a white-noise process. When $\mathbf{J}$ is diagonal but not equal to the identity, $\mathbf{f}$ is nonstationary and referred to as a colored-noise process. When the samples of $\mathbf{f}$ are independent random variables, $\mathbf{K}$ will be diagonal, and if full incoherence is defined in terms of the diagonality of $\mathbf{K}$, it follows that $\mathbf{f}$ is an incoherent field. On the other hand, if full incoherence is defined in terms of the diagonality of $\mathbf{J}$, then $\mathbf{f}$ must be a zero-mean process for $\mathbf{J}$ to also be diagonal and thus $\mathbf{f}$ is an incoherent field. When the samples are independent and identically distributed, $\mathbf{K}$ will equal the identity matrix, leading to similar consequences, but with the additional feature that we now have a stationary field.

\section{B. Full Coherence}

Now we consider coherent fields. Any two samples of such a field must be fully correlated. As discussed above, this means that the magnitude of their normalized correlation has the largest possible value of unity. Therefore the elements of the complex coherence matrix $\mathbf{L}$ must have unit magnitude:

A discrete optical field is fully coherent if all elements of the associated normalized mutual intensity matrix (complex coherence matrix) have unit magnitude, i.e.,

$$
|L(m, n)|=1, \quad m, n=1, \ldots, N,
$$

or, alternatively, if the associated mutual intensity matrix J has unit rank.

We have already seen that in this case the matrices $\mathbf{J}$ and $\mathbf{L}$ both have unit rank, are of outer-product form, and consequently have only one nonzero eigenvalue (property 6 in Sections 2 and 3 and the following discussion). The sole nonzero eigenvalue of $\mathbf{L}$ is equal to $N$. (This is easily 
deduced from the fact that for any square matrix the sum of the eigenvalues is equal to the trace: the sum of the diagonal elements.)

Full coherence can be alternatively defined through the requirement that the rank of $\mathbf{J}$ or $\mathbf{L}$ equal unity, or that $\mathbf{J}$ or $\mathbf{L}$ have only one nonzero eigenvalue, or that $\mathbf{J}$ or $\mathbf{L}$ be of outer-product form.

If we denote the outer-product form of the $\mathbf{L}$ matrix of a coherent field as $\mathbf{L}=\mathbf{u u}^{\mathrm{H}}$, we see that all elements of $\mathbf{u}$ are also of unit magnitude; that is, the normalized correlation corresponds to the unnormalized correlation of a field normalized to unit magnitude. This means that the result of the normalization is to remove the effects of spatial intensity variation from the field. This is desirable, since we are more interested in the relative correlations of pairs of points than in the variation of intensity across the field.

Deterministic fields (fields that are not random processes) are coherent. Since the ensemble average is superfluous, the matrix $\mathbf{J}$ for such a field is of outer-product form and hence the field is coherent. On the other hand, not every coherent field is necessarily deterministic.

\section{Stationarity}

Another common concept that can be characterized in terms of the matrices defined is stationarity. Here we restrict our attention to second-order stationarity, which means that second-order correlations depend only on the separation of the two points in question and not on their absolute positions. As is common practice, we define stationarity for a finite domain based on periodic boundary conditions:

A discrete optical field is stationary if the associated mutual intensity matrix is circulant:

$$
J(m, n)=J((m-n) \bmod N), \quad m, n=1, \ldots, N ;
$$

that is, if the elements on any given circular diagonal are equal to each other. A circular diagonal is one that wraps around in the sense of periodic boundary conditions. If $\mathbf{J}$ is circulant, so is $\mathbf{L}$. An alternative approach would be to define stationarity by requiring that $\mathbf{J}$ be Toeplitz instead of circulant when circular diagonals are replaced with ordinary diagonals. However, it is standard practice to work with circulant matrices in such discrete settings: they are not only more suitable for analytical formulations but also in fact easier to relate to a continuous framework.

We conclude this section with the continuous counterparts of some of the results presented in Subsections 4.A4.C. An optical field is fully incoherent if the associated complex coherence function is zero except when $x_{1}=x_{2}$ :

$$
L\left(x_{1}, x_{2}\right)=\delta\left(x_{1}-x_{2}\right) / \delta(0),
$$

which is equal to unity when $x_{1}=x_{2}$ by definition [Eq. (14)]. The eigenfunctions of $L\left(x_{1}, x_{2}\right)$ are $u_{\nu}(x)=\delta(x$ $-\nu$ ), and its eigenvalues are all equal to each other: $\lambda(\nu)=1 / \delta(0)$. With regard to the other limit, an optical field is fully coherent if the associated complex coherence function has unit magnitude for all $x_{1}$ and $x_{2}$ :

$$
\left|L\left(x_{1}, x_{2}\right)\right|=1 \text {. }
$$

In this case, the eigenfunctions satisfy $\left|u_{\nu}(x)\right|=1$, and there is only one nonzero eigenvalue: $\lambda(\nu)=\delta(\nu$ $-\nu_{0}$ ), as discussed in Section 2. We note that the "eigenvalue mass" $\int_{-\infty}^{\infty} \lambda(\nu) \mathrm{d} \nu$ for both the fully incoherent case $\left(\int_{-\infty}^{\infty}[1 / \delta(0)] \mathrm{d} \nu\right)$ and the fully coherent case $\left[\int_{-\infty}^{\infty} \delta\left(\nu-\nu_{0}\right) \mathrm{d} \nu\right]$ is equal to unity. [The first of these follows from the identity $\int_{-\infty}^{\infty} \exp (i 2 \pi \nu x) \mathrm{d} \nu=\delta(x)$ with $x=0$, whereas the second follows from the definition of the delta function.] It is more generally possible to show that $\int_{-\infty}^{\infty} \lambda(\nu) \mathrm{d} \nu=1$ for the eigenvalues of all complex coherence functions $L\left(x_{1}, x_{2}\right)$; the total eigenvalue mass is always equal to unity. [This can be shown by setting $x_{1}=x_{2}=x$ in Eq. (11), integrating both sides with respect to $x$, and using Eq. (10).] In the fully incoherent case, the unit eigenvalue mass is spread as uniformly and thinly as possible over all values of $\nu$. On the other hand, in the fully coherent case, the unit eigenvalue mass is concentrated as much as possible at a single point. These idealizations are of course just as unphysical as perfect delta functions and sinusoids of infinite extent; in reality, the eigenvalue mass will be neither spread out over an infinite extent nor concentrated at a single point. Nevertheless, these idealizations serve as useful formal devices representing limiting cases. Finally, before we leave this section, we note that an optical field is considered stationary if $J\left(x_{1}, x_{2}\right)=J\left(x_{1}-x_{2}\right)$.

\section{DEGREE OF PARTIAL COHERENCE OF A FIELD}

Having established the two extremes of full coherence and full incoherence and provided precise definitions for them in terms of their normalized correlation matrices, we now define scalar measures of the degree of partial coherence of a field. This can be accomplished by interpolating any of the characteristics of the matrices in question. For instance, we saw that the eigenvalues of the matrix $\mathbf{L}$ for incoherent fields are all equal to unity, whereas only one of the eigenvalues of a coherent field is nonzero. Thus a suitable function of the eigenvalues that takes its extremes at these two special cases can serve as a measure of the degree of partial coherence. Alternatively, we saw that the matrix $\mathbf{L}$ for an incoherent field is diagonal, representing maximum concentration around the diagonal, whereas the same matrix for a coherent field has elements with unit magnitude, representing maximum spread. Again, a suitable interpolation will yield a measure of coherence.

There are many ways of constructing such interpolation functions, leading to several definitions of such a measure, of which we can show some to be identical, some monotonically related, and some quite different. Here we will present some of the more obvious candidates for such a measure. Our purpose here is not to offer an exhaustive analysis of these but to motivate the different possibilities.

All of these measures $c^{\prime}$ are defined such that their minimum value $c_{\text {min }}^{\prime}$ corresponds to full incoherence and 
their maximum value $c_{\max }^{\prime}$ corresponds to full coherence (or the other way around). We will employ the following mapping to obtain a final measure $c$, which equals zero for incoherent light and equals unity for coherent light:

$$
c=\frac{c^{\prime}-c_{\text {incoh }}^{\prime}}{c_{\text {coher }}^{\prime}-c_{\text {incoh }}^{\prime}},
$$

where $c_{\text {incoh }}^{\prime}$ and $c_{\text {coher }}^{\prime}$ are the extreme values corresponding to the incoherent and coherent cases $\left(c_{\text {incoh }}^{\prime}=c_{\min }^{\prime}\right.$ and $c_{\text {coher }}^{\prime}=c_{\text {max }}^{\prime}$, or the other way around).

\section{A. Eigenvalue-Based Measures}

As stated above, incoherent light is characterized by all unity eigenvalues and coherent light by one nonzero eigenvalue of the matrix $\mathbf{L}$. We also recall that the eigenvalues are all nonnegative and that their sum is equal to $N$.

Definition 1. Based on the observation above, the more concentrated the eigenvalues are around the largest one, the more coherent the light, and the more uniformly spread they are, the more incoherent the light. It will be convenient to assume that in the general case the eigenvalues are arranged in decreasing order. Therefore the following measure of the spread of the eigenvalues away from the largest eigenvalue (which has index $n=1$ ) will serve as a measure of the degree of partial coherence:

$$
c_{1}^{\prime}=\frac{1}{N} \sum_{n=1}^{N}(n-1)^{2} \lambda_{n} .
$$

When all eigenvalues are unity (incoherent light), we have $c_{1}^{\prime}=(N-1)(2 N-1) / 6$, and when only one eigenvalue is nonzero (coherent light), we have $c_{1}^{\prime}=0$.

Definition 2. If we think of the distribution of eigenvalues as a function of the index $n$ as constituting a kind of generalized spectral distribution, then the above measure essentially corresponds to a spectral spread. The distribution of values of the eigenvalues can also be measured without reference to a particular discrete variable with respect to which they are indexed, and merely as the spread among a group of numbers; that is, as the variance of the eigenvalues:

$$
c_{2}^{\prime}=\frac{1}{N} \sum_{n=1}^{N}\left(\lambda_{n}-1\right)^{2},
$$

where the 1 subtracted from $\lambda_{n}$ is the average value of the eigenvalues. When all eigenvalues are unity, we have $c_{2}^{\prime}=0$, and when only one eigenvalue is nonzero, we have $c_{2}^{\prime}=N-1$.

Definition 3. The concept of measuring maximal concentration versus maximally uniform spread brings to mind the concept of entropy, which measures maximum order versus disorder. Therefore the following alternative to the previous measure naturally asserts itself:

$$
c_{3}^{\prime}=-\sum_{n=1}^{N} \frac{\lambda_{n}}{N} \log \left(\frac{\lambda_{n}}{N}\right),
$$

where we recall that $\sum_{n=1}^{N}\left(\lambda_{n} / N\right)=1$. The base of the logarithm is of no consequence. When all eigenvalues are unity, we have $c_{3}^{\prime}=\log N$, and when only one eigenvalue is nonzero, we have $c_{3}^{\prime}=0$ ( since $z \log z \rightarrow 0$ as $z \rightarrow 0$ ).

A similar measure was previously proposed in Refs. 9 and 16 , based on the eigenvalues of the unnormalized mutual intensity matrix $\mathbf{J}$ rather than the normalized complex coherence matrix $\mathbf{L}$ that we are employing. Without normalization, this measure does not properly characterize the incoherent limit. Our formulation based on normalized matrices solves this and other problems associated with the mutual intensity matrix.

Although space does not permit us to further discuss the relationships between these measures in this paper, we note that Definitions 2 and 3 (and also Definition 5, to be given in Subsection 5.B) can be shown to be monotonically related to each other.

\section{B. Matrix Spread-Based Measures}

We now turn our attention from the eigenvalues to the complex coherence matrix $\mathbf{L}$ itself. When $\mathbf{L}$ is diagonal, the light is incoherent, and when $\mathbf{L}$ has all unitmagnitude entries, the light is coherent. Interpolation between these two extremes leads to the following measures of partial coherence.

Definition 4. We consider the spatial variance (moment of inertia) around the diagonal of the matrix $\mathbf{L}$. Since $|L(m, n)|=|L(n, m)|$ from Hermitian symmetry and assuming periodic boundary conditions so that $L(m, N+n)=L(m, n)$ and $L(N+m, n)=L(m, n)$, we can form the following measure of spread:

$$
c_{4}^{\prime}=\sum_{n=1}^{N} \sum_{m=1}^{N}(m-n)^{2}|L(m, n)|^{2} .
$$

When $\mathbf{L}$ is the unit matrix (incoherent light), we have $c_{4}^{\prime}=0$, and when all elements of $\mathbf{L}$ have unit magnitude (coherent light), we have $c_{4}^{\prime}=2 \Sigma_{l=1}^{N}(N-l) l^{2}$. It should be noted that $c_{4}^{\prime}$ is not strictly a spatial variance, since we do not normalize by $\sum_{n=1}^{N} \sum_{m=1}^{N}|L(m, n)|^{2}$. Alternatively, to be truer to the spirit of periodic boundary conditions, it is possible to replace $(m-n)^{2}$ with $(N-m+n)^{2}$ when $m-n>N / 2$.

Definition 5. Alternatively, we can ignore the spatial distribution of the matrix entries and simply measure the energy of the matrix $\mathbf{L}$. Recalling that elements of $\mathbf{L}$ are always less than or equal to unity and that the diagonal elements are always equal to unity, we can see that this measure will be minimum for the unit matrix (incoherent light) and maximum when all elements have unit magnitude (coherent light):

$$
c_{5}^{\prime}=\frac{1}{N^{2}} \sum_{n=1}^{N} \sum_{m=1}^{N}|L(m, n)|^{2} .
$$

When $\mathbf{L}$ is the unit matrix, we have $c_{5}^{\prime}=1 / N$, and when all elements of $\mathbf{L}$ have unit magnitude, we have $c_{5}^{\prime}=1$.

These and other measures of partial coherence will be further studied and compared in another paper. Here we satisfy ourselves by noting that there is no definition that is clearly superior in all circumstances; different measures are appropriate for different situations. (For instance, consider the question of whether a mutual inten- 
sity function with a narrow mainlobe and sidelobes, or one with a wider mainlobe but no sidelobes, is more coherent.)

We conclude this section by presenting the continuous versions of some of the measures of partial coherence discussed in Subsections 5.A and 5.B. Recall that we always have $\lambda(\nu) \geqslant 0$ and $\int_{-\infty}^{\infty} \lambda(\nu) \mathrm{d} \nu=1$ and that full incoherence corresponds to the case where the eigenvalue mass is spread uniformly over the $\nu$ axis and full coherence corresponds to the case where the eigenvalue mass is concentrated at a single point. First, we consider the continuous counterpart of Definition 2. Consider the quantity $\int_{-\infty}^{\infty} \lambda^{2}(\nu) \mathrm{d} \nu$. In the fully incoherent limit, this integral evaluates to $1 / \delta(0)=0$. As we approach the fully coherent limit, this integral tends to $\delta(0)=\infty$. To map this to a measure taking values in the interval $[0,1]$, we employ the inverse tangent function (other suitable smooth functions may also be used). Therefore our measure takes the form

$$
c_{2}=\frac{2}{\pi} \arctan \left[\int_{-\infty}^{\infty} \lambda^{2}(\nu) \mathrm{d} \nu\right],
$$

which is zero for full incoherence, is unity for full coherence, and takes on intermediate values for general partially coherent fields. Now let us consider the continuous counterpart of Definition 3. The definition of entropy that is the underlying motivation of this definition is of the form $-\Sigma_{k} p_{k} \log p_{k}$, where $p_{k}$ are probabilities such that $\Sigma_{k} p_{k}=1$. The continuous counterpart of this definition of entropy is $-\int p(\nu) \log p(\nu) \mathrm{d} \nu$ such that $\int p(\nu) \mathrm{d} \nu$ $=1$. Since $\int_{-\infty}^{\infty} \lambda(\nu) \mathrm{d} \nu=1$ and $\lambda(\nu) \geqslant 0$, the distribution of the eigenvalue mass can be interpreted as a probability density function, and therefore we can form the quantity $-\int_{-\infty}^{\infty} \lambda(\nu) \log \lambda(\nu) \mathrm{d} \nu$ based on the same motivation as that in the discrete case. This quantity tends to plus infinity for the fully incoherent limit and to minus infinity for the fully coherent limit. Therefore we define our measure as

$$
c_{3}=\frac{1}{\pi} \arctan \left[\int_{-\infty}^{\infty} \lambda(\nu) \log \lambda(\nu) \mathrm{d} \nu\right]+\frac{1}{2},
$$

which again is zero for full incoherence, is unity for full coherence, and takes on intermediate values in the general case. For concreteness, the base of the logarithm may be chosen as $e$. Although not presented here, expressions for the continuous versions of the spread-based measures can also be written.

\section{FOURIER-DOMAIN ANALYSIS}

In Section 4, we stated the definitions of coherence and incoherence in terms of autocorrelations (or autocovariances) of the original field $\mathbf{f}$. Here we wish to state the equivalent conditions for coherence and incoherence in the Fourier domain.

Let us denote the discrete Fourier transform of the discrete optical field f by $\widetilde{\mathbf{f}}=\mathbf{F f}$, where $\mathbf{F}$ is the $N \times N$ unitary discrete Fourier transform matrix defined by $F(m, n)=N^{-1 / 2} \exp (-i 2 \pi m n / N)$.

Let us find the mutual intensity matrix $\mathbf{J}_{\mathbf{f}}$ of the Fourier transform of the optical field:

$$
\mathbf{J}_{\tilde{\mathbf{f}}}=\left\langle\mathbf{F f}(\mathbf{F f})^{\mathrm{H}}\right\rangle=\mathbf{F} \mathbf{J}_{\mathbf{f}} \mathbf{F}^{\mathrm{H}} .
$$

We observe that the mutual intensity of the Fourier transform is the double Fourier transform of the mutual intensity of the original.

We know that a circulant $\mathbf{J}$ matrix corresponds to a stationary random process. (If the matrices in question represented the kernel of a linear system, a circulant matrix would represent a linear-shift-invariant system.) Complex harmonics are known to be the eigenvectors of circulant matrices. Therefore the discrete Fourier transform matrix, whose columns are the complex harmonics, will diagonalize any circulant matrix ${ }^{5}$.

$$
\Lambda_{\mathbf{J}}=\mathbf{F} \mathbf{J F}^{\mathrm{H}} .
$$

First, let us assume that $\mathbf{J}_{\mathbf{f}}$ is circulant, corresponding to a stationary optical field. Then $\mathbf{J}_{\mathbf{f}}$ will be diagonal. In other words, a stationary optical field is characterized by a circulant matrix in the space domain but by a diagonal matrix in the Fourier domain. Now let us assume that $\mathbf{J}_{\mathbf{f}}$ is diagonal, corresponding to an incoherent optical field. Then $\mathbf{J}_{\tilde{\mathbf{f}}}$ will be circulant. In other words, an incoherent optical field is characterized by a diagonal matrix in the space domain but by a circulant matrix in the Fourier domain. We may summarize by saying that incoherence and stationarity are Fourier duals (or conjugates).

If we assume that $\mathbf{J}_{\mathbf{f}}$ is of unit rank, then it is possible to show that $\mathbf{J}_{\tilde{f}}$ is also of unit rank. In other words, a coherent field is represented by a unit-rank matrix in both the space and Fourier domains. Since matrices of this form represent coherent fields, we may say that coherence is its own Fourier dual. (This is also consistent with the fact that if a field is deterministic, its Fourier transform will also be deterministic.)

If we restrict our attention only to second-order stationarity and do not pay attention to a possible nonstationarity in the mean, the circulantness of $\mathbf{K}$ can be taken as an alternative definition of stationarity. In this case, the above discussion can also be taken to be valid for $\mathbf{K}$. However, it should be noted that $\mathbf{K}_{\tilde{\mathbf{f}}} \neq \mathbf{F K}_{\mathbf{f}} \mathbf{F}^{\mathrm{H}}$.

\section{YOUNG'S EXPERIMENT AND CORRELATION VERSUS COVARIANCE}

The purpose of this section is to consider the superposition of two random variables, which may represent two samples of an optical field, and discuss how the intensity of the superposed field depends on the correlation or the covariance of these two random variables. This will lead us to discuss the use of correlation ( $\mathbf{J}$ or $\mathbf{L}$ ) versus that of covariance (K or $\mathbf{M}$ ) in characterizing partially coherent fields.

Traditionally, the concepts of spatial coherence and incoherence are motivated in terms of the visibility of fringes in Young's experiment under quasimonochromatic conditions. The visibility is usually defined as the ratio of the difference between the maximum and minimum intensities to the sum of the maximum and minimum intensities. Incoherent light is associated with 
zero fringe visibility, whereas coherent light is associated with maximum fringe visibility. Maximum fringe visibility corresponds to maximum constructive/destructive interference, where the two complex amplitudes are added or subtracted with full force. Zero visibility corresponds to complete lack of interference, where the intensities are added without any cross terms. Therefore our traditional understanding of coherence and incoherence has to do with the ability or the lack of ability to constructively or destructively interfere.

Let the complex amplitude of light represented by these two random variables be denoted by $f_{1}$ $=A_{1} \exp \left(i \phi_{1}\right)$ and $f_{2}=A_{2} \exp \left(i \phi_{2}\right)$, where $A_{1}, A_{2}, \phi_{1}$, and $\phi_{2}$ are real. The total observed field is $f=f_{1}$ $+f_{2} \exp (i \alpha)$, where $\alpha$ is the phase difference associated with the path-length difference in Young's experiment. Let us further denote the ensemble-averaged mean of $f_{1}$ by $\mu_{1}$ and that of $f_{2}$ by $\mu_{2}$. Then we can write $f_{1}=\mu_{1}$ $+g_{1}$ and similarly for $f_{2}$, where $g_{1}$ is a zero-mean random variable. Thus any nonzero-mean random variable can be interpreted as the sum of a nonrandom number and a zero-mean random variable. The intensity $I$ $=\left\langle\left|f_{1}+f_{2} \exp (i \alpha)\right|^{2}\right\rangle$ resulting from the superposition of $f_{1}$ and $f_{2}$ can be written in two alternative but equal forms:

$$
\begin{aligned}
I= & I_{1}+I_{2}+2 \sqrt{I_{1} I_{2}}\left|L_{12}\right| \cos \left[\angle\left(L_{12}\right)-\alpha\right] \\
=\mid & \left.\mu_{1}\right|^{2}+P_{1}+\left|\mu_{2}\right|^{2}+P_{2} \\
& +2 \sqrt{P_{1} P_{2}}\left|\mu_{1} \mu_{2}^{*} / \sqrt{P_{1} P_{2}}+M_{12}\right| \\
& \times \cos \left[\angle\left(\mu_{1} \mu_{2}^{*} / \sqrt{P_{1} P_{2}}+M_{12}\right)-\alpha\right],
\end{aligned}
$$

where $P_{i}=\left\langle\left|g_{i}\right|^{2}\right\rangle, \quad I_{i}=\left\langle\left|f_{i}\right|^{2}\right\rangle, \quad$ and thus $I_{i}=\left|\mu_{i}\right|^{2}$ $+P_{i}$. We use $\angle(z)$ to denote the argument of $z$. In deriving the second of these expressions, we used a trigonometric formula for the sum of two cosines. The first of these expressions has been expressed in terms of the normalized non-mean-subtracted (correlation) L-matrix entry $L_{12}=\left\langle f_{1} f_{2}^{*}\right\rangle / \sqrt{I_{1} I_{2}}$, and the second has been expressed in terms of the normalized mean-subtracted (covariance) M-matrix entry $M_{12}=\left\langle g_{1} g_{2}^{*}\right\rangle / \sqrt{P_{1} P_{2}}$. The above expressions take their extreme values over $\alpha$ (in terms of which visibility is defined) when the cosine term is equal to \pm 1 . Thus the visibility, defined as the ratio $(\max -\min ) /(\max +\min )$, is given by

$$
\begin{aligned}
\text { Visibility } & =\frac{2 \sqrt{I_{1} I_{2}}\left|L_{12}\right|}{I_{1}+I_{2}} \\
& =\frac{2 \sqrt{P_{1} P_{2}}\left|\mu_{1} \mu_{2}^{*} / \sqrt{P_{1} P_{2}}+M_{12}\right|}{\left|\mu_{1}\right|^{2}+P_{1}+\left|\mu_{2}\right|^{2}+P_{2}} .
\end{aligned}
$$

When the means $\mu_{1}$ and $\mu_{2}$ are equal to zero, both of the above expressions become identical. In this case, $I_{i}=P_{i}$ and $M_{12}=L_{12}$. In the case of incoherent light, we expect intensity addition and no cross terms (zero visibility), implying that $\left|L_{12}\right|=\left|M_{12}\right|=0$. In the case of coherent light, we expect complex-amplitude addition (maximum visibility), implying that $\left|L_{12}\right|=\left|M_{12}\right|=1$. Therefore we see that the mathematical definitions of co- herence and incoherence offered in Section 4 are consistent with the traditional understanding of these concepts when the means are zero.

Now we turn our attention to the general nonzeromean case, when Eqs. (30) and (31) [or Eqs. (32) and (33)] are no longer identical. Intensity addition (no interference) means that the resultant intensity is given by $I_{1}$ $+I_{2}$ and therefore that the visibility is zero. If we associate incoherence with intensity addition and zero visibility, it follows that $\left|L_{12}\right|=0$, but the same cannot be said for $\left|M_{12}\right|$. If $\left|M_{12}\right|=0$, the resultant intensity is given by $I_{1}+I_{2}+2\left|\mu_{1} \mu_{2}\right| \cos \left[\angle\left(\mu_{1} \mu_{2}^{*}\right)-\alpha\right]$. We observe that although the correlation of the mean-subtracted part is zero, the deterministic parts corresponding to the nonzero means lead to an interference term that violates strict intensity addition. (In other words, the mean field behaves like a deterministic coherent component, so that a field with nonzero mean cannot be strictly incoherent if we associate incoherence with intensity addition.) However, if we choose to define incoherence with the alternative concept of intensity addition of the mean-subtracted parts only ( $P=P_{1}+P_{2}$ ), treating the means as mere insignificant biases, then for this definition of incoherence it follows that $\left|M_{12}\right|=0$ but that $\left|L_{12}\right| \neq 0$.

Complex-amplitude addition (full interference) means that the extremes of the resultant intensity are given by $I_{1}+I_{2} \pm 2 \sqrt{I_{1} I_{2}}$ and that the visibility is $2 \sqrt{I_{1} I_{2}} /\left(I_{1}\right.$ $+I_{2}$ ). If we associate coherence with complexamplitude addition and maximum visibility, it follows that $\left|L_{12}\right|=1$, but the same cannot be said for $\left|M_{12}\right|$. If $\left|M_{12}\right|=1$, the normalized correlation of the meansubtracted parts is unity, but as a whole, complexamplitude addition is violated. However, if we choose to define coherence with the alternative concept of complexamplitude addition of the mean-subtracted parts only ( $P=P_{1}+P_{2} \pm 2 \sqrt{P_{1} P_{2}}$ ), treating the means as mere insignificant biases, then for this definition of coherence it follows that $\left|M_{12}\right|=1$ but that $\left|L_{12}\right| \neq 1$.

In summary, we have shown that defining coherence and incoherence in terms of the normalized complex coherence matrix $\mathbf{L}$ is appropriate if we expect incoherence to be characterized by intensity addition and coherence to be characterized by complex-amplitude addition of the whole fields, including any nonzero-mean components. On the other hand, it is appropriate to use the meansubtracted matrix $\mathbf{M}$ if the means of the fields are treated as insignificant biases and are ignored. The use of the mean-subtracted $\mathbf{K}$ and $\mathbf{M}$ matrices is more consistent with the definition of the correlation coefficient of two random variables as commonly used in statistics and probability theory. ${ }^{11}$ On the other hand, the mutual intensity matrix $\mathbf{J}$ and the complex coherence matrix $\mathbf{L}$ are more established in optics.

\section{CONCLUSION}

In this paper, we set the foundations of a linear algebraic theory of partial coherence. While containing no new physics, the presented formulation allows precise definitions of concepts such as coherence and incoherence, offers new insights, and allows us to make the most of the conceptual and algebraic tools of linear algebra. This pa- 
per was mainly formulated in the discrete notation of vectors and matrices, but continuous versions of many results and relations were also presented.

We offered several definitions for the degree of partial coherence of a light field $c$ such that $c=0$ for full incoherence and $c=1$ for full coherence. A complete study of the various alternatives and their comparison will be the subject of another paper.

We carefully discussed Young's experiment to clearly show the relationship between our physical understanding of the concept of coherence and the mathematical definitions presented in this paper. In particular, we discussed the relative merits of using correlation or covariance functions as the basis for our definitions.

We believe that the formulation presented will be especially useful in optical information processing applications, since it will allow the precise analytical and numerical formulation of such problems. In particular, in a further paper we will discuss how the present formalism can be applied to the problem of synthesizing light with desired mutual intensity distributions from light with given mutual intensity (previous attempts at dealing with this problem include Refs. 17-20). It may also be of interest to explore the relationship of the quantities discussed in this paper to measures of beam quality as applied to partially coherent beams. ${ }^{21}$

The corresponding author, Haldun M. Ozaktas, may be reached by e-mail, haldun@ee.bilkent.edu.tr.

\section{REFERENCES AND NOTES}

1. L. Mandel and E. Wolf, Optical Coherence and Quantum Optics (Cambridge U. Press, Cambridge, UK, 1995).

2. M. Born and E. Wolf, Principles of Optics, 6th ed. (Pergamon, Oxford, UK, 1980).

3. J. W. Goodman, Statistical Optics (Wiley, New York, 1985).

4. J. Perina, Coherence of Light (Van Nostrand Reinhold, London, 1971).

5. G. Strang, Linear Algebra and Its Applications, 3rd ed. (Harcourt Brace Jovanovitch, San Diego, Calif., 1988).

6. A. V. Oppenheim, R. W. Schafer, and J. R. Buck, Discrete
Time Signal Processing, 2nd ed. (Prentice-Hall, Englewood Cliffs, N.J., 1999).

7. A. W. Lohmann, R. G. Dorsch, D. Mendlovic, Z. Zalevsky, and C. Ferreira, "Space-bandwidth product of optical signals and systems," J. Opt. Soc. Am. A 13, 470-473 (1996).

8. R. N. Bracewell, "Radio interferometry of discrete sources," Proc. IRE 46, 97-105 (1958).

9. H. Gamo, "Matrix treatment of partial coherence," in Progress in Optics, E. Wolf, ed. (North-Holland, Amsterdam, 1964), Vol. 3, pp. 187-332.

10. H. M. Ozaktas, Z. Zalevsky, and M. A. Kutay, "Sampling and the number of degrees of freedom," in The Fractional Fourier Transform with Applications in Optics and Signal Processing (Wiley, New York, 2001), Sec. 3.3.

11. A. Papoulis, Probability, Random Variables, and Stochastic Processes, 3rd ed. (McGraw-Hill, New York, 1991).

12. T. Habashy, A. T. Friberg, and E. Wolf, "Application of the coherent-mode representation to a class of inverse source problems," Inverse Probl. 13, 47-61 (1997).

13. B. Zhang and B. Lu, "Transformation of Gaussian Schellmodel beams and their coherent-mode representation," J. Opt. 27, 99-103 (1996).

14. The formal analogy between the discrete and continuous cases is discussed in many texts; for instance, see C. CohenTannoudji, B. Diu, and F. Laloë, Quantum Mechanics (Wiley, New York, 1977), 2 vols.

15. B. E. A. Saleh and M. C. Teich, Fundamentals of Photonics (Wiley, New York, 1991).

16. H. Gamo, "Intensity matrix and degree of coherence," J. Opt. Soc. Am. 47, 976 (1957).

17. M. F. Erden, H. M. Ozaktas, and D. Mendlovic, "Synthesis of mutual intensity distributions using the fractional Fourier transform," Opt. Commun. 125, 288-301 (1996).

18. M. F. Erden, "Repeated filtering in consecutive fractional Fourier domains," Ph.D. thesis (Bilkent University, Ankara, Turkey, 1997).

19. M. A. Kutay, "Generalized filtering configurations with applications in digital and optical signal and image processing," Ph.D. thesis (Bilkent University, Ankara, Turkey, 1999).

20. M. A. Kutay, H. M. Ozaktas, M. F. Erden, and S. Yüksel, "Discrete matrix model for synthesis of mutual intensity functions," in Optical Processing and Computing: A Tribute to Adolf Lohmann, D. P. Casasent, H. J. Caulfield, W. J. Dallas, and H. H. Szu, eds., Proc. SPIE 4392, 87-98 (2001).

21. T. D. Visser, A. T. Friberg, and E. Wolf, "Phase-space inequality for partially coherent optical beams," Opt. Commun. 187, 1-6 (2001). 